\title{
A 59-Year-Old Female Presenting With Bloating and Flatulence: Is the Intestinal Gas Volume in Plain Abdominal Radiographs Correlated With Symptoms?
}

\author{
Jie-Hyun Kim, MD, Jung Soo Park, MD, Young Hoon Yoon, MD, Hyojin Park, MD* and Sang In Lee, MD \\ Department of Internal Medicine, Institute of Gastroenterology, Gangnam Severance Hospital, Yonsei University College of Medicine, Seoul, \\ Korea
}

A 59-year-old woman with bloating and flatulence for 6 months visited to our hospital. Her symptoms did not improve after treatment with antiflatulents, prokinetics and probiotics. A plain abdominal radiograph showed a moderate amount of in- testinal gas (Fig. 1A). The intestinal gas volume was measured in the plain radiograph using the imaging software Image-pro Plus ${ }^{\circledR}$ (Media Cybernetics Inc, Bethesda, MD, USA). ${ }^{1}$ To standardize the quantity of intestinal gas, the ratio of the quantity of intestinal
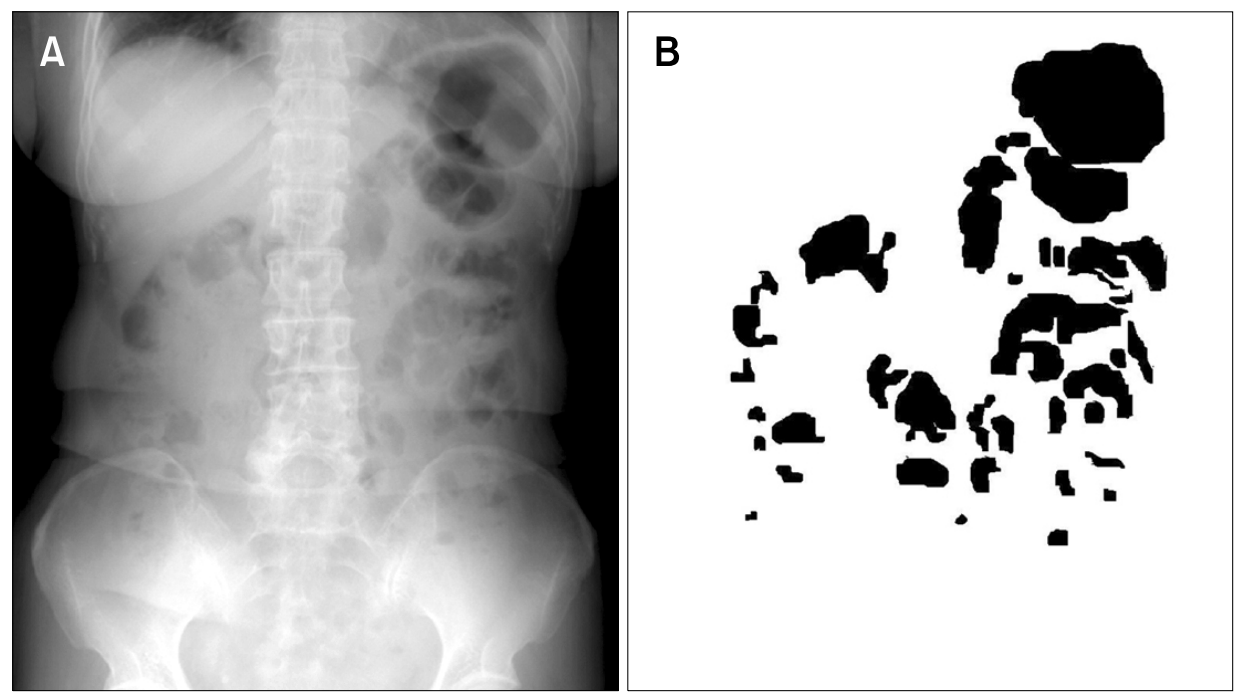

Figure 1. The plain abdominal radiograph shows a moderate amount of intestinal gas (A), which has decreased after treatment with an antibiotic (C). The gas volume score (GVS) is measured in the plain abdominal radiograph using imaging software (B, D). The black area shows bowel gas and the black border is the abdominal background. The GVS is calculated as the area of bowel gas divided by the area of background. The GVS decreased from 0.131 (B) to 0.049 (D) following treatment with antibiotic.

Received: June 18, 2010 Revised: June 25, 2010 Accepted: June 28, 2010

(c) This is an Open Access article distributed under the terms of the Creative Commons Attribution Non-Commercial License (http://creativecommons. org/licenses/by-nc/3.0) which permits unrestricted non-commercial use, distribution, and reproduction in any medium, provided the original work is properly cited.

*Correspondence: Hyojin Park, MD

Department of Internal Medicine, Institute of Gastroenterology, Gangnam Severance Hospital, Yonsei University College of Medicine, 712 Eonju-ro, Gangnam-gu, Seoul 135-720, Korea

Tel: +82-2-2019-3318, Fax: +82-2-3463-3882, E-mail: HJPARK21@yuhs.ac

Financial support: None.

Conflicts of interest: None. 

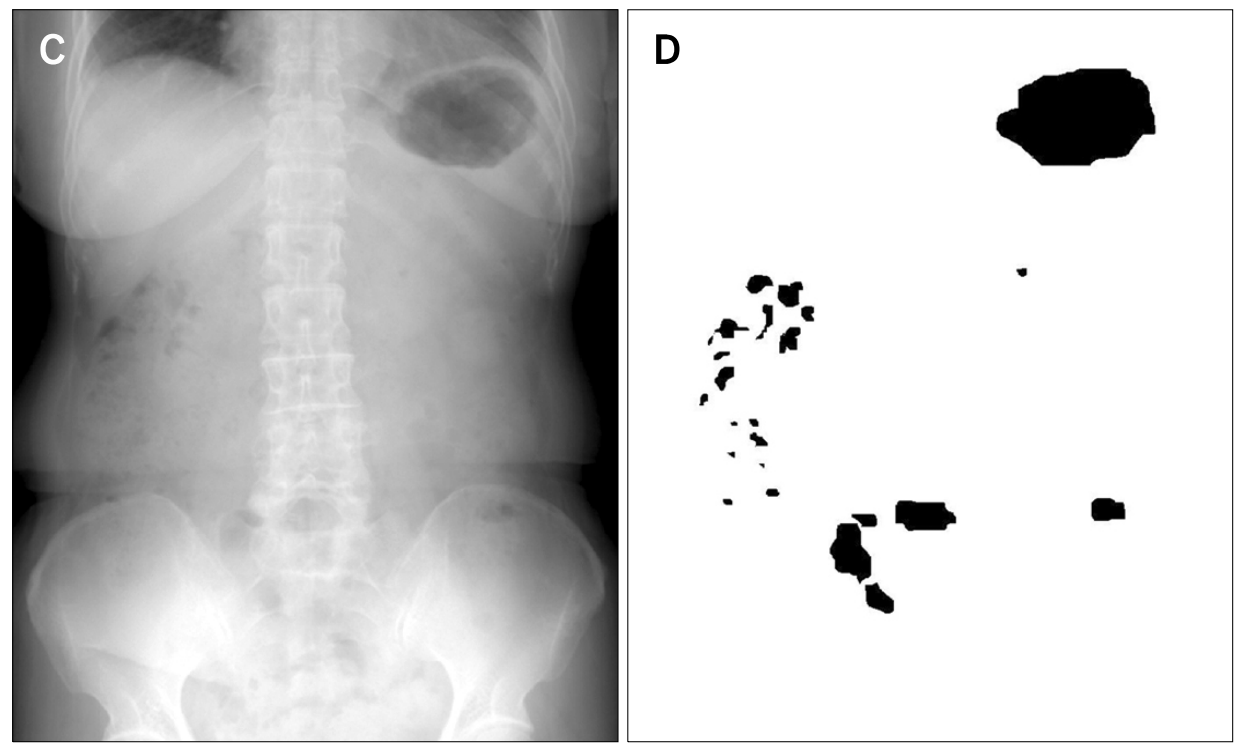

Figure 1. Continued.

gas to the pixel value in the region surrounded by a horizontal line tangential to the suprasymphyseal margin, a horizontal line tangential to the uppermost point of the diaphragm, and the lateralmost lines tangential to the right and left costal arches was defined as the gas volume score. ${ }^{2,3}$ The gas volume score was calculated at 0.131 (Fig. 1B). Since the other medications had not been effective for the bloating, an antibiotics (metronidazole) was started to treat possible intestinal bacterial overgrowth (however, generally rifaximin is used for intestinal bacterial overgrowth). After 2 weeks, her symptoms had disappeared. When the gas volume score was measured in a follow-up plain abdominal radiograph, the amount of intestinal gas in the plain abdominal radiograph had decreased (Fig. 1C) and the gas volume score was lower at 0.049 (Fig. 1D).

The amount of intestinal gas in plain abdominal radiographs may be correlated with bloating symptoms. Moreover measuring the intestinal gas volume might be more useful when combined with a breath test for intestinal bacterial overgrowth.

\section{References}

1. Kim YG, Moon JT, Lee KM, Chon NR, Park H. The effects of probiotics on symptoms of irritable bowel syndrome. Korean J Gastroenterol 2006;47:413-419.

2. Morken MH, Berstad AE, Nysaeter G, Berstad A. Intestinal gas in plain abdominal radiographs does not correlate with symptoms after lactulose challenge. Eur J Gastroenterol Hepatol 2007;19:589-593.

3. Koide A, Yamaguchi T, Odaka T, et al. Quantitative analysis of bowel gas using plain abdominal radiograph in patients with irritable bowel syndrome. Am J Gastroenterol 2000;95:1735-1741. 